\title{
Modulation of flexible filaments dynamics due to attachment angle relative to the flow
}

DOI:

10.1016/j.expthermflusci.2018.11.015

\section{Document Version}

Accepted author manuscript

Link to publication record in Manchester Research Explorer

\section{Citation for published version (APA):}

Silva Leon, J., \& Cioncolini, A. (2019). Modulation of flexible filaments dynamics due to attachment angle relative to the flow. Experimental Thermal and Fluid Science, 102, 232-244.

https://doi.org/10.1016/j.expthermflusci.2018.11.015

\section{Published in:}

Experimental Thermal and Fluid Science

\section{Citing this paper}

Please note that where the full-text provided on Manchester Research Explorer is the Author Accepted Manuscript or Proof version this may differ from the final Published version. If citing, it is advised that you check and use the publisher's definitive version.

\section{General rights}

Copyright and moral rights for the publications made accessible in the Research Explorer are retained by the authors and/or other copyright owners and it is a condition of accessing publications that users recognise and abide by the legal requirements associated with these rights.

\section{Takedown policy}

If you believe that this document breaches copyright please refer to the University of Manchester's Takedown Procedures [http://man.ac.uk/04Y6Bo] or contact uml.scholarlycommunications@manchester.ac.uk providing relevant details, so we can investigate your claim.

\section{OPEN ACCESS}




\title{
Modulation of flexible filaments dynamics due to attachment angle relative to the flow
}

\author{
Jorge Silva-Leon ${ }^{1,2}$ and Andrea Cioncolini ${ }^{1}$ \\ ${ }^{1}$ School of Mechanical, Aerospace and Civil Engineering, University of Manchester, George Begg Building, \\ Sackville Street, M1 3BB, Manchester, United Kingdom \\ ${ }^{2}$ Escuela Superior Politécnica del Litoral, ESPOL, Facultad de Ingeniería en Mecánica y Ciencias de la \\ Producción, Campus Gustavo Galindo Km 30.5 Vía Perimetral, P.O. Box 09-01-5863, Guayaquil, Ecuador
}

This paper describes experiments carried out in a wind tunnel with three flexible silicone filaments (length to diameter ratio $L / D=50,100,150$ ) hanging in crossflow in the range of reduced velocities of $7<U^{*}<150$ and at various attachment angles $\left(0 \leq \alpha \leq 90^{\circ}\right)$ with respect to the flow direction. At low reduced velocities, due to the negligible bending stiffness, the filaments were statically reconfigured but remained mostly rectilinear along their lengths, except for the relatively small bent portion of the filaments close to the upstream fixed end. As the reduced velocity was further increased the filaments started vibrating, but in certain cases large-amplitude flapping motions were observed. Despite of the negligible role of the attachment angle at the fixed end on the static reconfiguration of the filaments, it was found that it plays a crucial role on the dynamics of the flapping motions, as it determines whether the filaments exhibit large-amplitude limit-cycle oscillation dynamics or noisy small-amplitude vibrations.

Key words: large-amplitude motion, flutter, limit-cycle oscillation, flexible filaments, attachment angle, flexible fluid-structure interaction.

\section{Introduction}

During the last decade, the study of flexible filament-like structures in fluid flow has attracted the attention of researchers due to the possibility of energy harvesting through flow-induced vibrations [1], enhancement of heat transfer [2] and also for their use on hairy coatings for drag reduction of bluff bodies [3]. A hairy coating consists of a layer of filaments (or fibers) which statically or dynamically reconfigure under the action of fluid flow. When attached to the rear surface of a bluff body, the compliance of the coating allows manipulating the wake characteristics by reducing its size and altering the vortex shedding behavior from the substrate bluff body. Due to their low cost and weight, hairy coatings are an attractive option for achieving drag reduction and lift stabilization of bluff bodies. For instance, Favier et al. [3] performed simulations of hinged cilia (rigid fibers) placed on the downstream face of a two-dimensional circular cylinder (disk), and reported that the hairy coating helped reducing drag (by 15\%) and lift fluctuations (by 44\%) on the cylinder. They suggested that in order to understand and reproduce a realistic hairy coating, longer and flexible fibers should be investigated. They anticipated that long fibers could bring benefits for the drag reduction capabilities of the hairy coating due to the increased adaptability to the flow. However, optimal drag reduction also depends on several other geometrical and material properties of the fibers, e.g. diameter, material density, elasticity, coverage area, and packing density or porosity. Indeed, experiments with a hairy disk, coated with rubber filaments, in a soap film confirmed that for an optimized set of parameters the drag could be reduced by $17 \%$ [4]. However, further understanding of the performance of hairy coatings requires more fundamental studies on the effects that individual parameters have on the dynamics of the individual fibers/filaments.

In this regard, experimental studies on the motions and dynamics of flexible filaments have usually been performed on soap-films in axial flow aligned with gravity [5]-[7]. However, soap-films restrict the motion of filaments to a single plane and therefore cannot resolve three-dimensional dynamics 
at large flow velocities. Regarding three-dimensional studies, Schouveiler et al. [8] on the other hand, studied filaments in air cross flow in a wind tunnel (mass ratio $m^{*}=\rho_{s} / \rho_{f}=1000$, where $\rho_{s}$ is the structure density, i.e. the filament, while $\rho_{f}$ is the fluid density). In their experiments the filaments hanged horizontally and thus were sagged due to their own weight. When subjected to fluid flow the filaments lifted and at a certain critical speed they started to vibrate. Schouveiler et al [8] used an asymptotic analysis to study this onset of vibration and concluded that the motion was caused by a variation in the mean fluid forces acting on the filament. Recently, a study carried out by Silva-Leon et al [9] on filaments hanging vertically in crossflow $\left(m^{*}=1000\right)$ highlighted the importance of the filament mass-damping parameter on the dynamics. Short filaments (characterized by higher damping) exhibited vibrational motions at all the velocities tested, whereas longer filaments (characterized by lower damping) exhibited rich dynamics as the incoming flow speed was gradually increased: low-amplitude vibration, large-amplitude flutter and chaotic flutter. This is a notable difference with the results obtained by Schouveiler et al [8] who did not report large-amplitude flutter motions. The only significant difference between the experimental set ups of Schouveiler et al. [8] and Silva-Leon et al. [9] is the attachment angle of the flexible filaments, i.e. the inclination with respect to the incoming flow of the filament fixed end: horizontal in the former study, and vertical in the latter. This suggests that the attachment angle may have an important, and presently not investigated, effect on the dynamics of flexible filaments in flow, and is what motivated the present study. The objective of the present work was to explore the influence of the attachment angle on the flapping dynamics of flexible filaments hanging in crossflow. Our results indicate that the attachment angle plays a crucial role in determining whether a flexible filament will exhibit large-amplitude limit-cycle oscillations or small-amplitude random vibrations.

The paper is organized as follows. In section 1 we introduce the experimental setup and tests procedure. In Section 2 we describe the results, while on Section 3 we provide a discussion and interpretation of the results obtained. On Section 4 we provide our concluding remarks.

\section{Experiments and methods}

\subsection{Test pieces characterization}

The filaments were made of commercial silicone rubber (density $\rho_{s}=1000 \mathrm{~kg} / \mathrm{m}^{3}$ ), and were produced via 3D printing using an additive manufacturing system (model: 3-D Bioplotter; make: Envisiontec $\mathrm{GMBH}$ ). This material and manufacturing method were adopted in order to produce highly flexible filaments with smooth surface finishing, uniform circular cross-section (to within \pm 10 $\mu \mathrm{m}$ ) and no permanent deformations as they could be printed in straight paths with no curvatures or bends. The uniformity of the filaments diameter $(D=0.40 \pm 0.02 \mathrm{~mm})$ was verified via Scanning Electron Microscopy. The modulus of elasticity $(E=225 \pm 5 \mathrm{kPa})$, bending rigidity $(E I=(2.75 \pm 0.2)$ $\left.\times 10^{-13} \mathrm{~N} / \mathrm{m}^{2}\right)$ and Poisson ratio $(v=0.0101 \pm 0.0004)$ of the filaments were determined from preliminary tension tests and static deflection tests. The natural vibration frequencies of the filaments were determined via forced vibration tests, while the damping ratio was determined from free vibration tests in still air. A description of the experimental procedure for these characterization tests can be found in [9], [10]. Table 1 summarizes the geometrical and dynamical properties measured for the three filaments used in the present study. All the filaments posses the same diameter, but are of different lengths $L=20,40$ and $60 \mathrm{~mm}$ (measured to within $\pm 0.5 \mathrm{~mm}$ ), thus giving aspect ratios of $L / D=50,100$ and 150 respectively. These filaments are labelled in Table 1 and for the rest of this paper as filament 1,2 and 3 respectively. The natural vibration frequency of the first four modes of vibration ( $f_{n 1}$ to $f_{n 4}$ in Table 1) were measured for filaments 2 and 3 , while only the first three modes of vibration were resolvable for filament 1 . Higher modes could not be resolved because the filament displacement was too small. As can be seen in Table 1, the natural vibration frequency and the damping ratio decrease with increasing filament length. 
Table 1. Geometrical and dynamical properties of filaments tested

\begin{tabular}{lllllll}
\hline Filament No. & $L(\mathrm{~mm})$ & $f_{n 1}(\mathrm{~Hz})$ & $f_{n 2}(\mathrm{~Hz})$ & $f_{n 3}(\mathrm{~Hz})$ & $f_{n 4}(\mathrm{~Hz})$ & $\zeta$ \\
\hline 1 & 20.0 & 5.4 & 21.0 & 47.0 & - & 0.0533 \\
2 & 40.0 & 3.1 & 8.8 & 17.6 & 30.2 & 0.0295 \\
3 & 60.0 & 2.5 & 6.0 & 11.0 & 17.5 & 0.0204 \\
\hline
\end{tabular}

In order to place them inside the wind tunnel, the filaments were inserted coaxially into straight stainless steel cylindrical tubes (length: $120 \mathrm{~mm}$, external diameter $0.9 \mathrm{~mm}$, internal diameter: $0.66 \mathrm{~mm}$ ) and fixed with glue cement to the free end of the support tube so as to reproduce a cantilever boundary condition, as depicted in Fig. 1. The homogeneity of the connection was visually verified by rotating the support around its axis and checking that the configuration assumed by the hanging filament remained the same. The length of the support tube was chosen in order to position the filaments hanging at approximately mid-height inside the wind tunnel test section. The diameter and thickness of the support tube were chosen large enough to avoid any vibrations or bending during the tests. The support tube was secured to a support plate and then inserted from the top of the wind tunnel test section, as shown in Fig. 4. In order to systematically vary the attachment angle of the filaments with respect to the flow, a portion of the free end of the support tubes was bent into different angles $\alpha=90$ through $0^{\circ}$ in steps of $15^{\circ}$, where $\alpha=90^{\circ}$ corresponds to the support tube emerging perpendicular to the incoming flow direction while $\alpha=0^{\circ}$ means the bent portion of the support tube is parallel to the flow direction, see Fig. 1 . Three lengths for the bent portion of the support tube were tested $(15,50$ and $75 \mathrm{~mm})$. It was verified that the filament flapping behaviour (amplitude and critical velocity for onset of flapping) was not altered when testing the different lengths for the bent portion, but a length of $15 \mathrm{~mm}$ was finally selected as this was less prone to vibrations, i.e. longer bent portions exhibited vibrations at high flow velocities. During the tests the filaments usually hanged below the level of the support tube due to gravity. Nevertheless, the frequency content in the wake of the support was measured at the range of wind speeds tested in this study $(1.0<U<15.0 \mathrm{~m} / \mathrm{s})$. The vortex shedding frequency was above $10^{3} \mathrm{~Hz}$, whereas the filament dynamics were usually characterized by frequencies in the range of $4-40 \mathrm{~Hz}$. Moreover, it was verified that the support did not vibrate during the tests. Additionally, the natural vibration frequencies of the support tube were estimated via standard Euler-Bernoulli beam theory and were found to be 2-3 orders of magnitude larger than the frequencies of motion observed for the filaments. Consequently, due to the large difference in time scales any interference between the support and filament was disregarded.

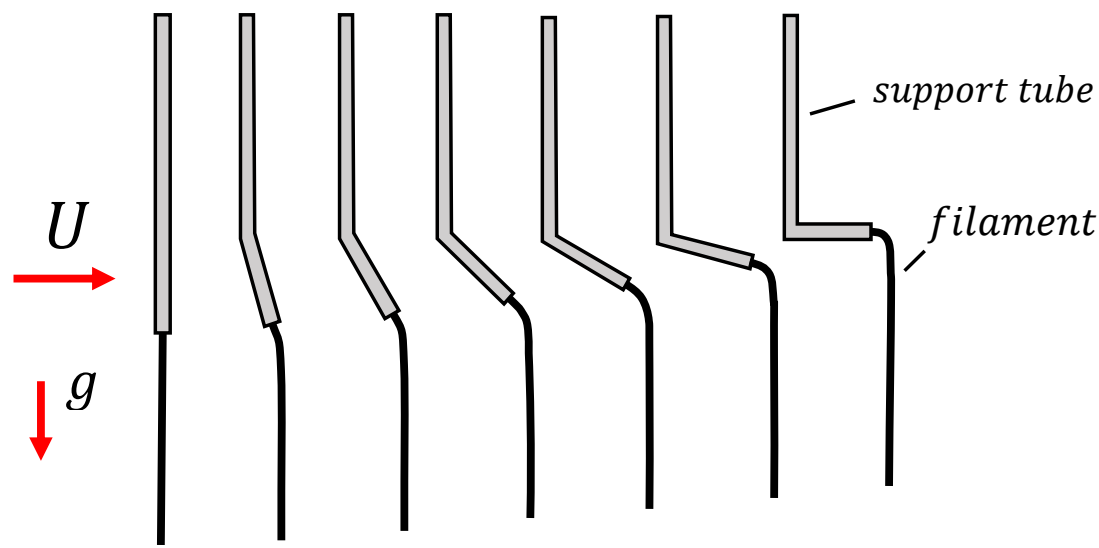

Figure 1. Schematic of support bent at different angles $\alpha$ with respect to the flow direction $(U)$. From left to right: $\alpha=90,75,60,45,30,15,0^{\circ}$. 


\subsection{Wind tunnel flow characterization}

The experiments were performed in an open-circuit wind tunnel (test section height and width of $350 \mathrm{~mm}$ ). The wind speed measurements were carried out with a calibrated hot-wire anemometer which was preliminarily validated in-situ via a standard experiment of vortex shedding from a circular cylinder in cross-flow (not reported here). The wind tunnel flow was characterized before the experiments to assure a uniform velocity profile across its test section and no interference due to boundary layers. The boundary layer extended less than $10 \mathrm{~mm}$ from the walls of the wind tunnel at a flow velocity of $0.4 \mathrm{~m} / \mathrm{s}$ corresponding to the lowest speed setting employed for this experiment, and the velocity profile across the wind tunnel was uniform to within $1 \%$ (excluding the boundary layer). The turbulence intensity was measured in the range of free stream velocities of $1 \mathrm{~m} / \mathrm{s}$ to $15 \mathrm{~m} / \mathrm{s}$ of interest here, and the results are shown in Fig. 2. As can be seen, the turbulence intensity increases linearly with wind speed until $U \approx 6.0 \mathrm{~m} / \mathrm{s}$, then slowly decreases with wind speed, with typical values on the order of $T u=0.5-0.7 \%$.

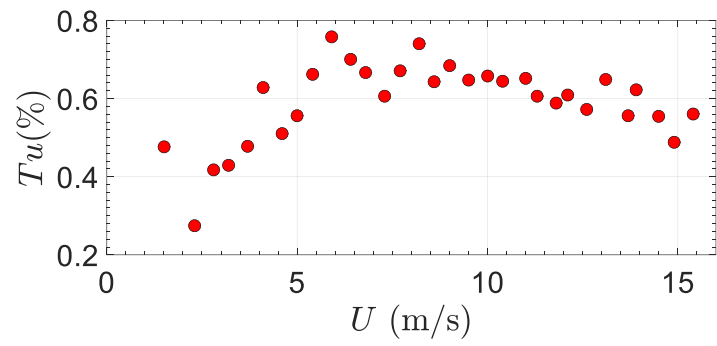

Figure 2. Turbulence intensity of the free-stream flow in the wind tunnel as function of the wind speed.

The flow was further characterized by estimating the integral and dissipation length scales (or macro- and micro scales, respectively) from 1 minute hot-wire velocity measurements (sampled at $20 \mathrm{kHz}$ ) of the turbulent flow. The macroscale $\left(L_{v x}\right)$ is an approximate measure of the size of the turbulent eddies in the flow that contain most of the turbulence energy [11]. On the other hand, the microscale $\left(\lambda_{v x}\right)$ is an approximate measure of eddies responsible for most of the dissipation of the turbulence energy in the flow. Assuming the flow is isotropic and homogeneous, an acceptable approximation in most engineering applications, these length scales may be estimated from the velocity power spectrum via Equations 1 and 2 shown below [11],

$$
\begin{gathered}
L_{v x}=\left[\frac{E(f) U}{4 \overline{u^{2}}}\right]_{f \rightarrow 0} \\
\lambda_{v x}=\left[\frac{2 \pi^{2}}{U^{2} \overline{u^{2}}} \int_{0}^{\infty} f^{2} E(f) \mathrm{d} f\right]^{-1}
\end{gathered}
$$

where $E(f)$ is the energy spectrum of the velocity signal as function of the frequency $f, U$ is the mean flow velocity, and $\bar{u}$ is the mean fluctuating velocity. In practice, for the macroscale the energy asymptote (as $f \rightarrow 0$ ) is determined from averaging the values of the energy spectrum at low frequencies. On the other hand, for the microscale the integration is done over the whole range of frequencies obtained. The results are shown in Fig. 3 where it can be seen that the macroscale lies in the range 5-60 $\mathrm{mm}$ while the microscale grows from 0.1 up to $1.5 \mathrm{~mm}$ in the range of wind speeds tested. As discussed later, the turbulence macroscale is crucial for the interpretation of the dynamics of lightly-damped structures. Regarding the turbulence intensity and the microscale, on the other hand, these are included to provide a thorough characterization of the system for future reference, in particular for the CFD community where a characterization of the flow turbulence is needed as input to the simulations. 

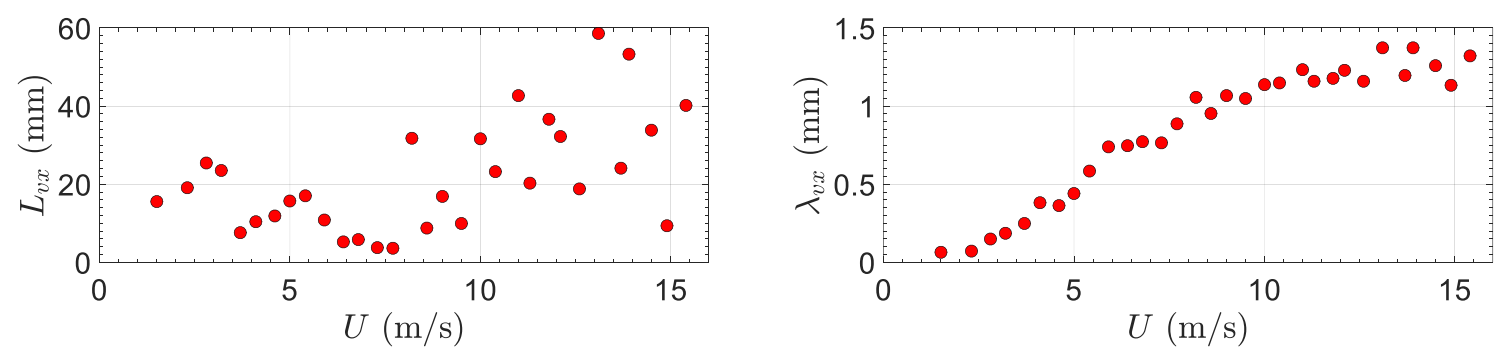

Figure 3. Estimated macroscale (left), and microscale (right) of the turbulent flow in the wind tunnel as function of the wind speed.

During the tests the atmospheric temperature and pressure in the laboratory were respectively 298 $\mathrm{K}$ and $101.6 \mathrm{kPa}$.

\subsection{Experimental procedure}

The tests began with the filament hanging sagged due to its own weight, then the wind speeds were increased gradually (steps of approximately $0.5 \mathrm{~m} / \mathrm{s}$ ) until a maximum of $15 \mathrm{~m} / \mathrm{s}$. The hotwire anemometer (HWA) was placed $200 \mathrm{~mm}$ downstream and $100 \mathrm{~mm}$ below the filament lowest point in order to avoid any contamination of the measurements due to the presence of the filament. When subjected to the steady flow, as depicted in Fig. 4, the flexible filament is lifted by the wind so it has a fixed upstream boundary condition and a free downstream end. The motion of the filaments was recorded via two high-speed cameras (Panasonic LUMIX-FZ200) located on top and side windows of the wind tunnel ( $X-Y$ and $X-Z$ planes) which provided a spatial resolution of $0.20 \mathrm{~mm} / \mathrm{pixel}$.

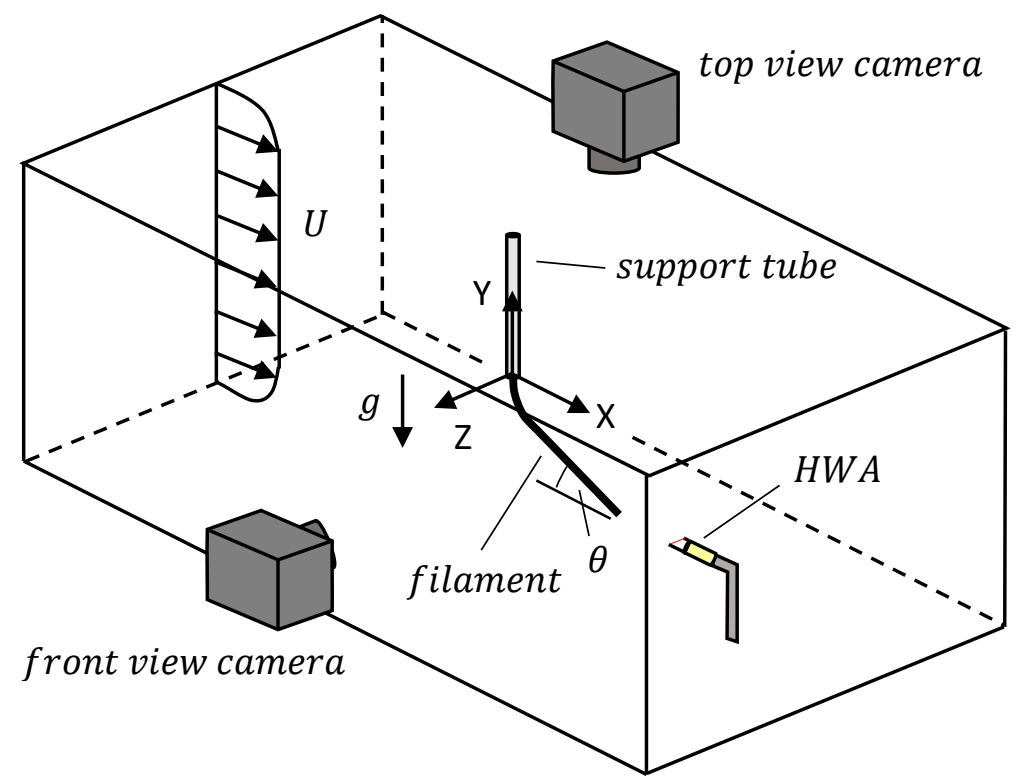

Figure 4. Schematic of experimental setup in wind tunnel test section.

The motions were recorded during 1 minute at 200 frames per second, and the videos were synchronized and post-processed via a purposely-written MATLAB script, and using the image processing toolbox of MATLAB.

Flow visualization, which would be quite useful in further characterizing the filaments dynamics, was not attempted due to technical difficulties. PIV measurements were not performed due to the high complexity of applying the technique for measuring the wake of a very thin filament (diameter 
of $0.4 \mathrm{~mm}$ ) which would produce a very fine structure that would be rapidly dissipated by the flow turbulence. This goes beyond our current capabilities. An alternative could be smoke-wire flow visualizations. We have tried this technique but unfortunately, this technique also has its limitations so wind speeds close to the onset of large-amplitude motion could not be employed as the smoke quickly dissipates. In the authors' opinion, high-fidelity CFD simulations would be valuable in characterizing the flow. In this respect, the data presented here will provide a useful benchmark test case.

\subsection{Data Analysis}

In order to justify the data analysis approach followed in this paper, we show in Fig. 5 an example of the behaviors of filament 3 when held at $\alpha=90^{\circ}$. In this figure we show the envelopes of motion in the $X-Y$ and $X-Z$ planes, as well as the trajectories of the free end of the filament as seen in the $Y-Z$ plane (corresponding to the view of an observer located downstream of the filament and facing upstream). The results shown correspond to two different wind speeds: a relatively low wind speed of $5.9 \mathrm{~m} / \mathrm{s}$ and the largest wind speed tested that is $15.4 \mathrm{~m} / \mathrm{s}$. As can be seen the motion is mostly two-dimensional (taking place in the $X-Y$ plane), but there is a non-negligible component of motion occurring in the $X-Z$ plane. Considering that the motion is not restricted to a plane we computed the instantaneous position of each point of the filament by considering the resultant displacement defined as:

$$
r_{y z}=\sqrt{y^{2}+z^{2}}
$$

Where $y$ and $z$ are the filament displacements observed in the $Y$ and $Z$ directions respectively. Consequently, the resultant displacement $r_{y z}$ combines the motion in two mutually orthogonal planes and can therefore be used to characterize both planar and out-of-plane motions such as those of interest here. For the purpose of the present paper we will only show the motion analysis of the filaments free end, as this point experiences the largest amplitude of motion (and thus higher signal-to-noise ratio) and, as confirmed in previous research, is also representative of the dynamics of any point along the filament [9].

At each wind speed the time history of displacement of the filament free end was studied via linear and nonlinear time-series analysis techniques. In particular, we obtained root-mean-squared values, autocorrelation functions, power spectra of frequency (PSD) and the trajectories in statespace (attractor). For the root-mean-squared values, the autocorrelation functions and PSDs the time-series were not previously filtered to remove noise. However, for obtaining smoother PSDs we employed the Welch averaging estimator [12] with a 600 sample Hamming window length and $50 \%$ overlap (built-in in MATLAB). We used the delayed vectors method [13] to reconstruct the system attractor in phase-space, whose topology provides useful qualitative characteristics of the filament dynamics that complete and corroborate the information obtained with standard timeseries analysis techniques. The concept of phase-space representation, rather than a classic analysis in time or frequency domain, is the key point in nonlinear time-series analysis. The topology of the trajectory in phase-space of a nonlinear system, in fact, provides important information regarding the fundamental dynamics of the systems being investigated. The problem is that in experimental studies one normally observes a time-series of scalar measurements of some quantity that depends on the current state of the system, and not the trajectory of the system in phase-space. The delayed vectors method allows reconstructing the trajectory of the system in phase-space from a time-series of scalar measurements, and can therefore be used in experimental studies such as the present one where only time-series of scalar measurements of some quantity (the filament free end displacement here) are available. This approach has been previously employed for studies of flapping flags and filaments in fluid flow [5], [9], [14]. The topology of the reconstructed attractors in state-space reveals useful qualitative indicators of the dynamics that complement the information obtained from standard time-series analysis techniques. In order to find appropriate embedding parameters (dimension $m$ and delay $\tau$ ) for the reconstruction of the attractors, we employed the mutual information and false nearest-neighbors algorithms available within the TISEAN free software package [15]. If appropriate embedding 
parameters are chosen, the reconstructed attractor is a faithful representation of the underlying dynamics of the system from which the time-series was obtained. For the purpose of attractor reconstruction the time series were filtered using the locally projective nonlinear noise reduction method included in the TISEAN package, which is specifically designed for nonlinear time series analysis and specifically takes into account the dynamics of the time series in phase-space. As specified in the TISEAN manual, time-series filtering is necessary for a successful attractor reconstruction because in certain cases the noise present in experimental data may obscure the topology of the reconstructed attractor.
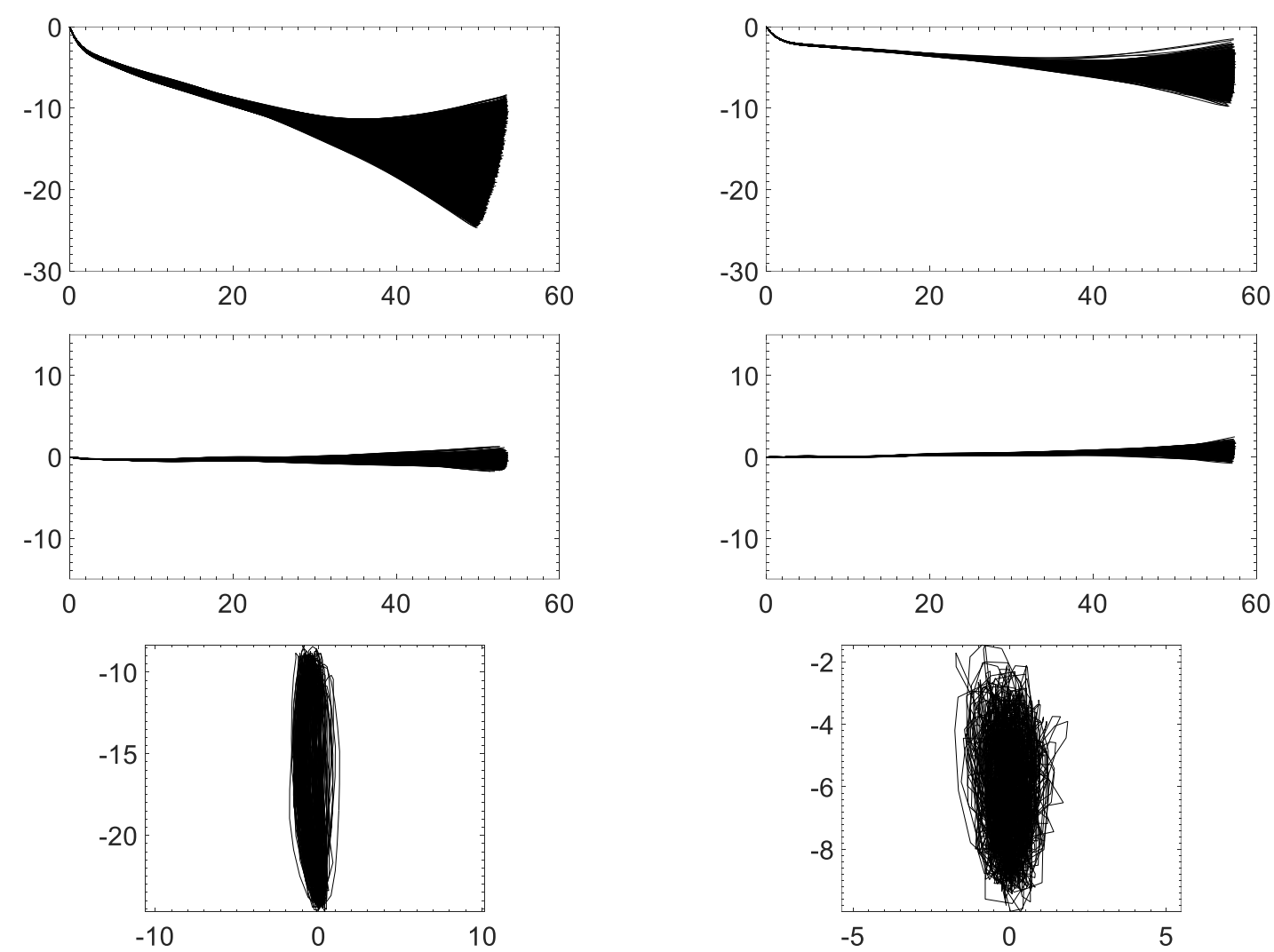

Figure 5. Raw results for filament 3 at two wind speeds (left column $U=5.9 \mathrm{~m} / \mathrm{s}$, right column $U=15.4 \mathrm{~m} / \mathrm{s}$ ), corresponding to 15 seconds of recordings (axes units in $\mathrm{mm}$ ): (top) envelopes of motion in X-Y plane; (middle) envelopes of motion in X-Z plane; (bottom) trajectories of filament free end in $Y-Z$ plane.

\section{Results and discussion}

\subsection{Filaments response}

Even though the motion of the filaments is three-dimensional, the most notable qualitative difference on the flapping behaviour can be appreciated in the X-Y plane. In Fig. 6 we show the envelopes of motion of the filaments when held at different attachment angles $\left(0 \leq \alpha \leq 90^{\circ}\right)$. In each of these plots the filament has been subjected to six selected wind speeds $(U=$ $0,1.5,2.5,3.5,4.5,7.5 \mathrm{~m} / \mathrm{s}$ ) so that each plot in Fig. 6 includes six envelopes of motion, one for each flow velocity. As will be explained later on, only four angles were tested for the shortest filament, while we considered that testing additional intermediate attachment angles was needed for better characterizing the behaviour of filaments 2 and 3 . The selected wind speeds were chosen in order to highlight two features of filament behaviour: the five lowest speeds to show the static 
reconfiguration and the highest wind speed was selected to exemplify a typical dynamic response. Irrespective of $\alpha$ in the case of filaments 2 and 3 the initial configurations at null wind speed are practically vertical due to their weight. As the wind speed is increased the filaments lift and remain in new static equilibrium positions. At the largest wind speed shown in this plot $(U=7.5 \mathrm{~m} / \mathrm{s})$ the filaments are already flapping. However, the amplitude of motion appears to be larger when $\alpha>$ $45^{\circ}$ compared to the cases where $\alpha<45^{\circ}$. On the other hand, filament 1 at $U=0 \mathrm{~m} / \mathrm{s}$ hangs vertical only for $\alpha=90^{\circ}$ whereas if it is hanged at smaller $\alpha$ the initial configuration appears more curved, thus evidencing the high stiffness of this specimen compared to its weight. Moreover, filament 1 appears practically static and just exhibits a low-amplitude vibration at the highest wind speed shown. In summary, depending on the attachment angle $\alpha$, the filaments appear to oscillate with a low-amplitude (see e.g. $\alpha=0^{\circ}$ ) or may incur into large-amplitude motions (see e.g. $\alpha=90^{\circ}$ ). Importantly, only filaments 2 and 3 undergo large-amplitude oscillations, and only for attachment angles above about $45^{\circ}$, while filament 1 does not undergo large-amplitude oscillations, at least in the range of reduced velocities employed here. This clearly shows that the filaments dynamics is modulated by the filament length, which controls the filament damping ratio, and also by the attachment angle: this latter effect was not systematically studied in previous research. This actually illustrates the basic qualitative difference between the results obtained on the experiments carried out by Schouveiler et al [8] for $\alpha=0^{\circ}$ and Silva-Leon et al [9] for $\alpha=90^{\circ}$ previously mentioned.

As can be noted in Fig. 6 during the static reconfiguration, filament 1 exhibits a variable curvature along its length. On the other hand, filaments 2 and 3 remain rectilinear along the majority of their lengths, except for the portion close to the support which of course is bent. In fact, for negligible bending rigidities the static reconfiguration angle $(\theta)$ of the rectilinear portion of filaments 2 and 3 can be predicted by the following correlation and definitions of the Cauchy $(\mathrm{Ca})$ and Buoyancy $(B)$ numbers [10]:

$$
\begin{gathered}
\theta=90 /\left(1+3.32(\mathrm{Ca} / B)^{1.33}\right)^{0.407} \\
C a=\frac{1}{2} \frac{\rho_{f} C_{d} D U^{2} L^{3}}{E I} \\
B=\frac{\left(\rho_{s}-\rho_{f}\right) g A L^{3}}{E I}
\end{gathered}
$$

where $\theta=90^{\circ}$ is a vertical inclination and $\theta=0^{\circ}$ is aligned with the horizontal (parallel to the incoming flow direction), $D$ stands for the filament diameter, $L$ is the axial length of the filament, $A$ is the filament axial cross section, $C_{d}$ is the drag coefficient of the vertical filament in cross flow (predicted here as explained in the Appendix), $U$ is the free stream flow speed, $g$ is the acceleration of gravity, $E I$ is the bending rigidity of the filament, while $\rho_{f}$ and $\rho_{s}$ are the densities of the fluid and the filament, respectively. The $C a / B$ then of course represents the ratio between the fluid drag force and the net weight of the filaments. The angle predictions from eq. (4) along with the experimental data points obtained from all the cases in which filaments 2 and 3 were static are shown in Fig. 7. Remarkably, there is an excellent agreement with the sigmoidal empirical correlation which is capable of predicting the saturating behavior of the data points at both extremes of $C a / B$. Importantly, besides providing validation for the experimental method, the results in Fig. 7 show that the attachment angle plays no role in the static reconfiguration of the filaments, because the measurements for different attachment angles all collapse onto the same trend line. As previously noted (Fig. 6) and better discussed later on, this is no longer the case when the filaments start moving, as their dynamic response is actually modulated by the attachment angle. 
$\alpha\left(^{\circ}\right)$

90

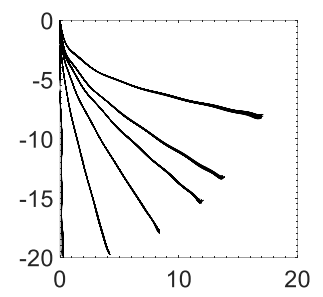

75

60

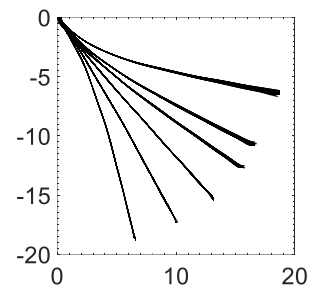

45

30

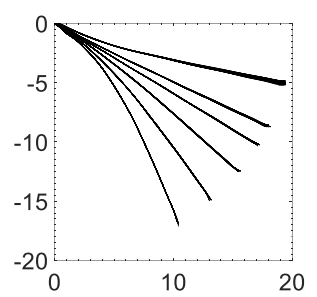

15

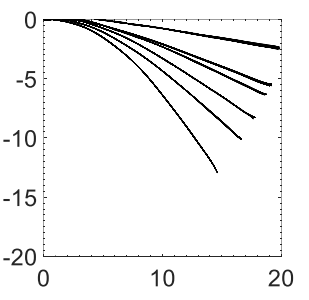

filament 2
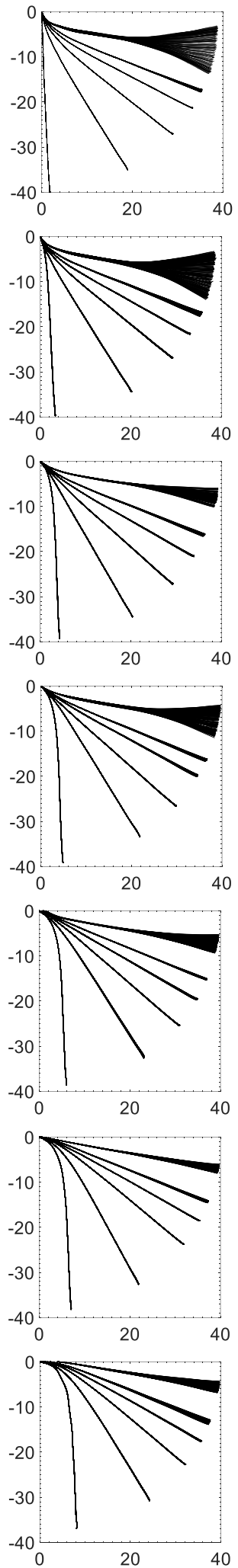

filament 3
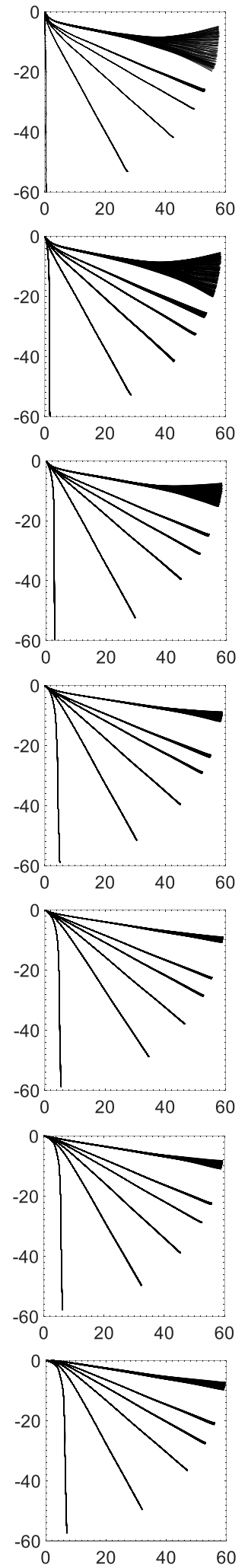

Figure 6. Envelopes of motion in the $X-Y$ plane for filaments 1-3 held at various attachment angles $\alpha$ and subjected to six wind speeds: $U=0,1.5,2.5,3.5,4.5,7.5 \mathrm{~m} / \mathrm{s}$. Axes units in $\mathrm{mm}$. Flow is from left to right. 

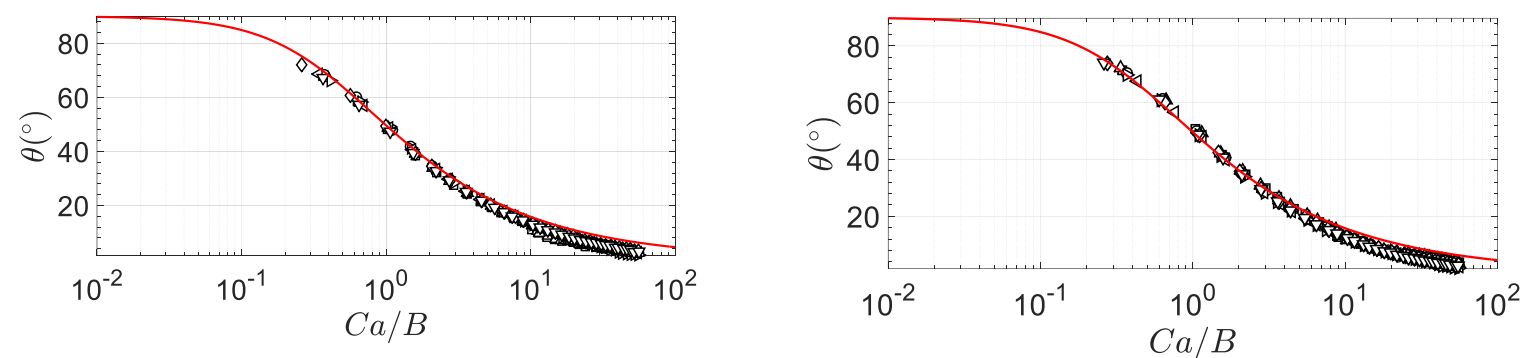

Figure 7. Equilibrium angle during the static reconfiguration of filament 2 (left) and filament 3 (right) at different attachment angles $\alpha: 90^{\circ}(\square), 75^{\circ}(\triangle), 60^{\circ}(\circ), 45^{\circ}(\triangleright), 30^{\circ}(\triangleleft), 15^{\circ}$ $(\diamond)$, and $0^{\circ}(\nabla)$. The solid red line is Eq. (4).

Beyond the static reconfiguration regime, as the wind speed is further increased the filaments start to oscillate. In order to compare the magnitude of these motions we obtained the amplitude of motion $\left(A_{y z}\right)$ by computing the root-mean-square (r.m.s.) value of the detrended displacement time-series $r_{y z}$ and then multiplied it times 2 to obtain the amplitude of a full excursion of the filament tip. The advantage of using the r.m.s. amplitude instead of other metrics (such as peak amplitude) as a measure of filament motion is its lower sensitivity to outliers or occasional bursts of large motion which may be present on a time-series. In Fig. 8 we show the results of the dimensionless amplitude of motion as function of the reduced wind velocity and attachment angle for filaments 1 through 3. The dimensionless amplitude response was defined as $A_{y z}{ }^{*}=A_{y z} / L$, where $A_{y z}$ is the r.m.s. amplitude of motion and $L$ is the length of the filament; and the reduced velocity defined as $U^{*}=U /\left(f_{n 1} L\right)$, where $U$ is the wind speed and $f_{n 1}$ is the first natural frequency of the filament from Table 1. The meaning of the color-coding of the symbols will be explained later. In the case of filament 1 , regardless of the attachment angle $\alpha$, the motion is initially null at low reduced velocities but then starts increasing gradually and proportionally to the reduced velocity. As can be noted, the amplitude of motion ranges between 1-6\% of the filament length and the amplitude reached is progressively smaller as the attachment angle is reduced from $\alpha=90^{\circ}$ to $0^{\circ}$. On the contrary, the attachment angle has a greater impact on the amplitude response of filament 2 and 3 . For these two filaments in particular, at low reduced velocities the amplitude first increases gradually. However, for attachment angles $\alpha>45^{\circ}$ the amplitude response curves exhibit an abrupt jump in magnitude which then gradually decreases at higher reduced velocities. On the other hand, for $\alpha<45^{\circ}$ the amplitude grows proportional to $U^{*}$ and does not exhibit a jump in the amplitude response, similar to the behaviour observed for filament 1 . Notably, the reduced velocity required for the onset of large-amplitude motion is larger for smaller $\alpha$. Moreover, it was verified that the onset of large-amplitude motion did not show a hysteresis cycle when the wind speed was reduced (not shown here). These peculiar differences in response are next studied in more detail by examining the dynamics of the filaments motion time-series. 

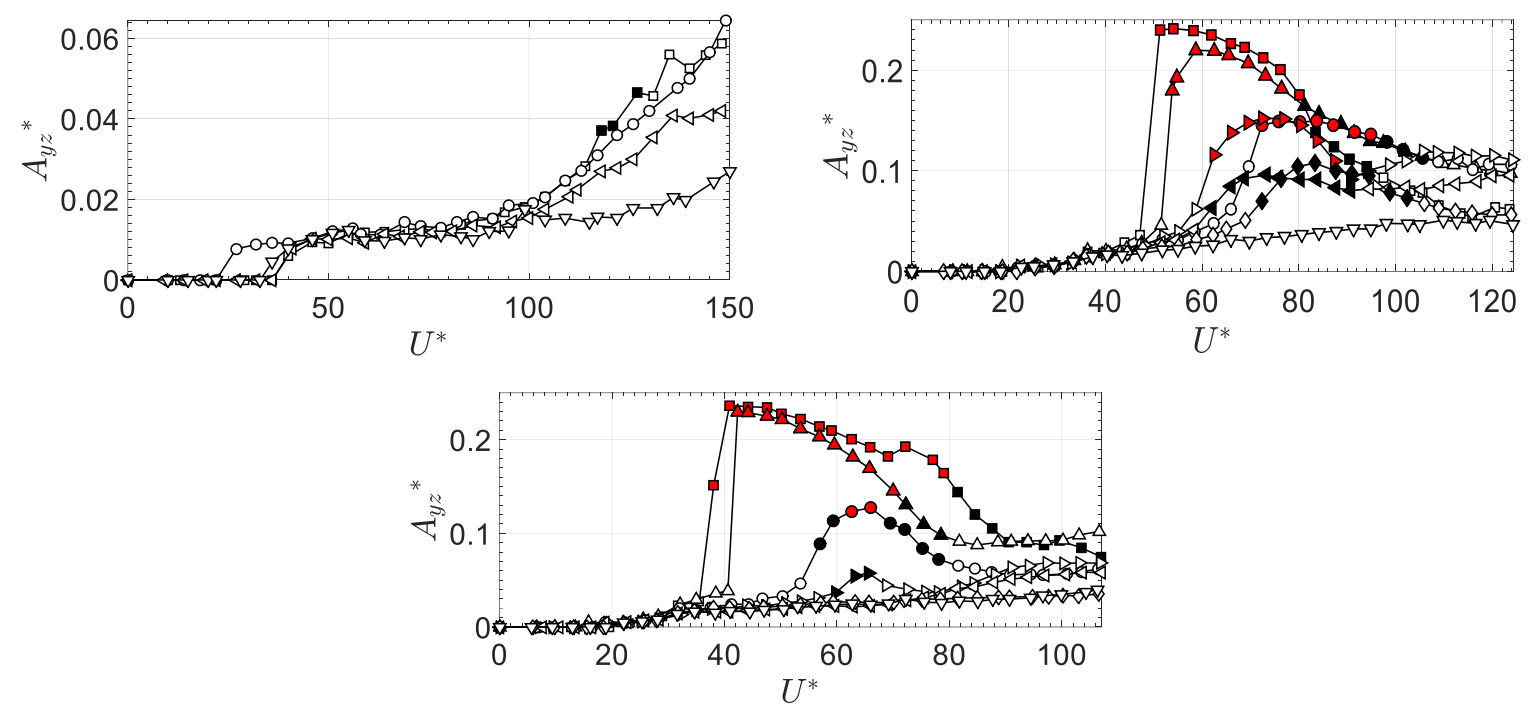

Figure 8. Dimensionless amplitude response vs reduced wind velocity of filament 1 (top left), filament 2 (top right) and filament 3 (bottom) at different attachment angles $\alpha: 90^{\circ}(\square)$, $75^{\circ}(\triangle), 60^{\circ}(\circ), 45^{\circ}(\triangleright), 30^{\circ}(\triangleleft), 15^{\circ}(\diamond)$, and $0^{\circ}(\nabla)$. Colour code explained in text.

\subsection{Time-series analysis of motion}

The dynamics analyses were carried out for the three filaments at each attachment angle for the whole range of reduced velocities tested. Nevertheless, for presentation purposes in the following we focus on selected representative results for filament 3 and for filament 1 . As will be explained later, these allow explaining the dynamics of filament 2 as well as the cases with different attachment angles. Complementary plots and the complete databank can be found in the Electronic Annex.

\subsubsection{Filament 3}

As was highlighted before, the amplitude-response of filament 3 is dependent on the attachment angle. Therefore we have chosen to show the results for $\alpha=90^{\circ}$ and $0^{\circ}$ which respectively are representative of the cases with and without abrupt jump in amplitude-response.

In Fig. 9 through 11 we show results for filament 3 when attached at $\alpha=90^{\circ}$. In Fig. 9 and 10 we show arrays of autocorrelation function plots and reconstructed attractors for the whole range of reduced velocities in which there was a resolvable amplitude of motion $\left(40<U^{*}<148\right)$, while power spectra for selected reduced velocities are presented in Fig. 11.

At reduced velocities below the onset of large-amplitude motion $\left(U^{*}<38\right)$ the autocorrelation functions reveal a fast decay which is an indication of high level of randomness in the signals. On the other hand, the attractors show that the dynamics change from a truly random motion characterized by an irregular blob in state-space $\left(U^{*} \leq 25\right)$ into a motion with a more regular structure which however covers the whole state-space which could denote that the motion has a broadband response due to intermittencies and noise $\left(25 \leq U^{*}<38\right)$. This is corroborated in Fig. 11 where the PSD for $U^{*}=23$ contains a single broadband peak with very high energy at the frequencies close to $0 \mathrm{~Hz}$, which is characteristic of chaotic dynamics in experimental systems [16]. Once a critical reduced velocity is reached (in this case $U^{*}=41$ ), the filament enters into a large-amplitude motion as was shown in Fig. 8. Notably, the autocorrelation function now decays slowly, the attractor has a clean ring-like shape in phase space and the PSD shows a prominent slender peak along with secondary peaks corresponding to harmonics of the dominant frequency. Evidently, the large-amplitude motion is characterized by a harmonic oscillation, which is also known as a limit-cycle oscillation (LCO). At higher velocities (Fig. 9) the autocorrelation functions decay more rapidly in time, and the attractors exhibit spread ring-like structures. However, the 
PSDs suggest that the motion gradually becomes broadband and no longer a period-1 but rather contain at least 2 non-multiple distinct frequencies (period-2 motion) with high energy levels at low frequencies. This shows that the limit-cycle oscillation dynamics are gradually corrupted and replaced by a more complex dynamics with a chaotic component. In these cases the autocorrelation function and PSD alone do not highlight the periodicity still existent in the timeseries. Instead, the attractor reflects this property through its topology (i.e. ring shape), although with intermittencies which explain its thicker structure. Therefore, we categorize these particular dynamics as an intermittent limit-cycle oscillation (ILCO).
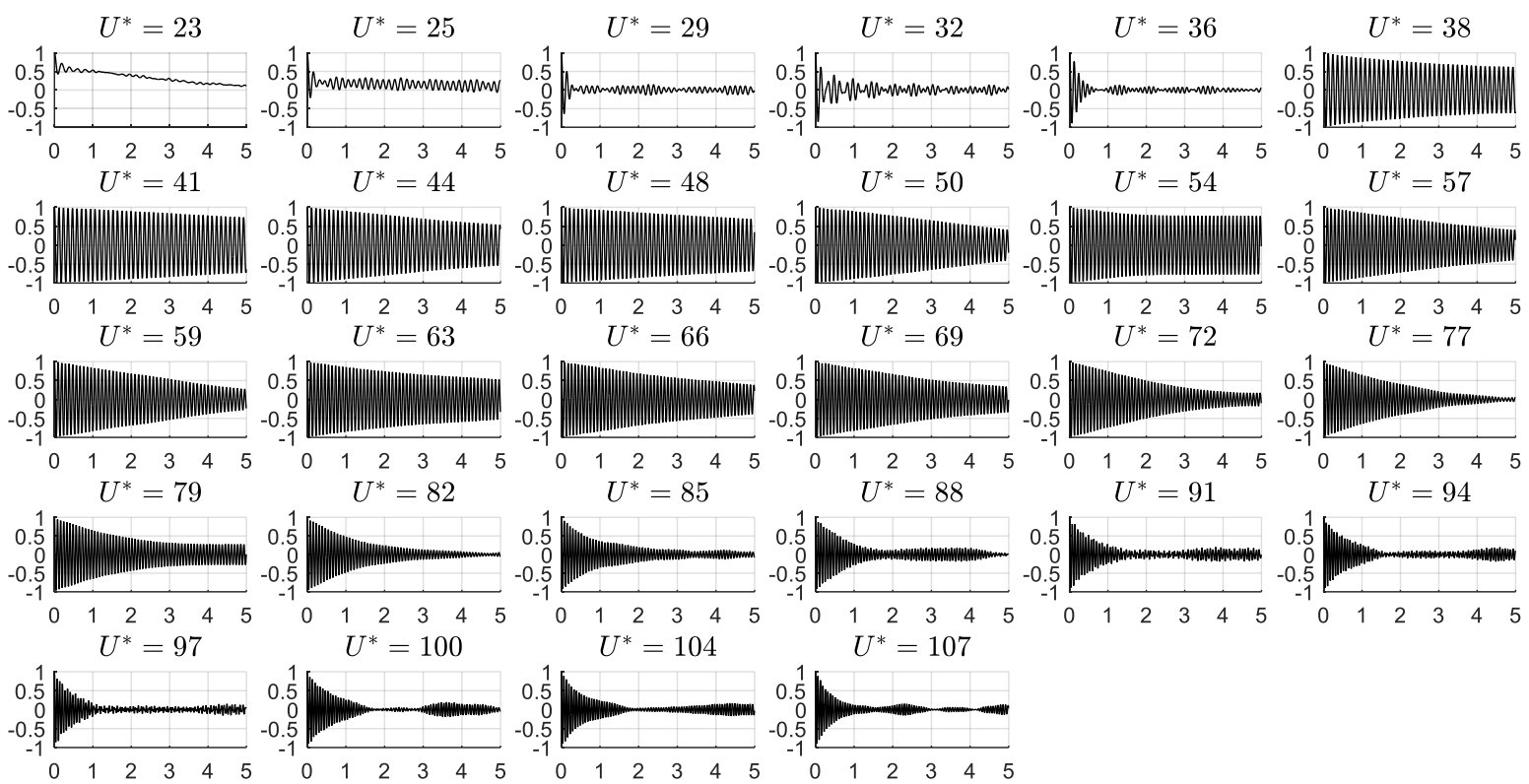

Figure 9. Autocorrelation function of the displacements of the free end of filament $3, \alpha=90^{\circ}$ (horizontal axis unit is $\mathrm{s}$ ).

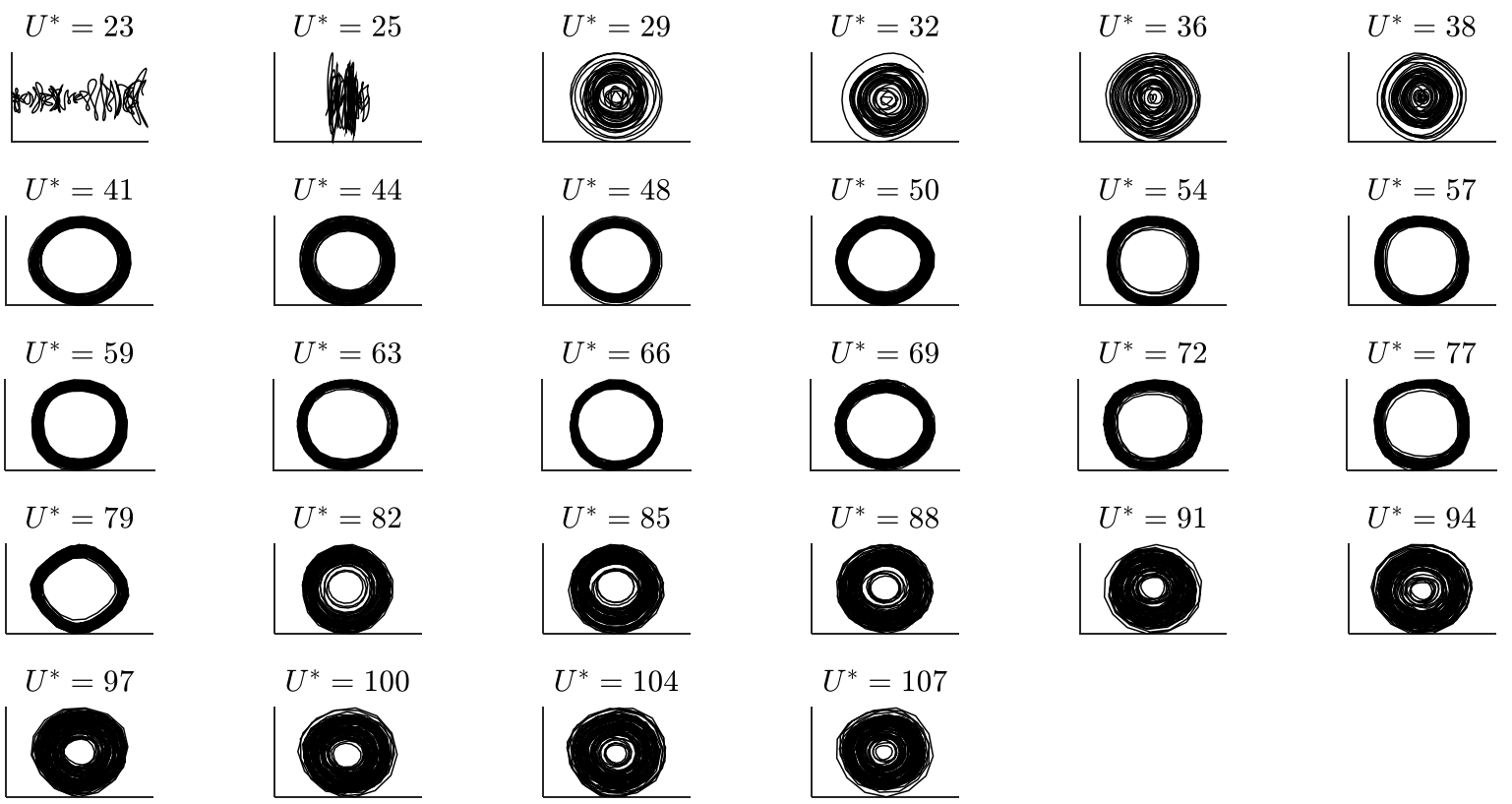

Figure 10. Reconstructed attractors in phase-space for the free end of filament $3, \alpha=90^{\circ}$. Horizontal axes: $r_{y z}(t)$, vertical axes: $r_{y z}(t+\tau)$. 

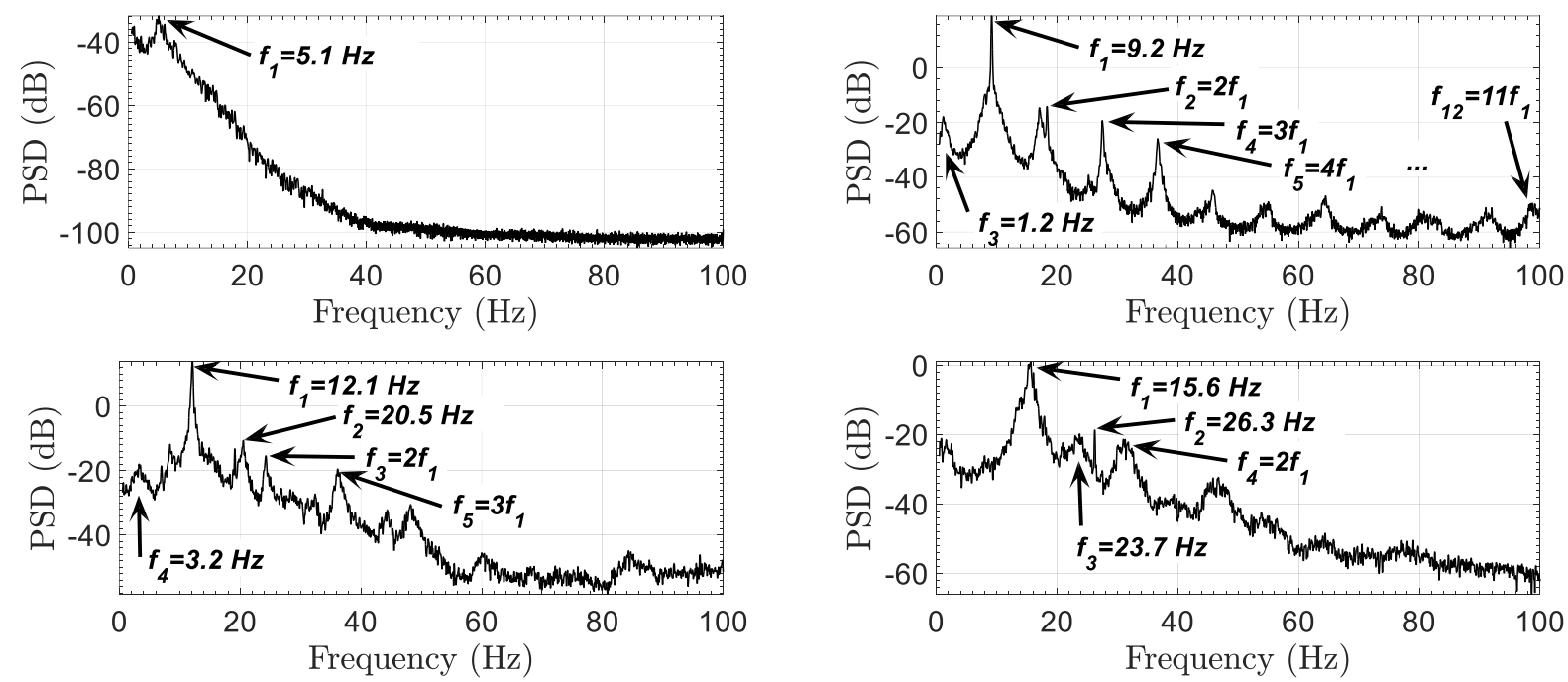

Figure 11. PSD of displacements of the free end of filament $3, \alpha=90^{\circ}$. (top left) $U^{*}=23$, (top right) $U^{*}=48$, (bottom left) $U^{*}=77$, (bottom right) $U^{*}=107$.

On the other hand, when filament 3 is held at $\alpha=0^{\circ}$, the results reflect a totally different progression of the dynamics as function of the reduced velocity (see Fig. 12 through 14). For the whole range of reduced velocities the correlation of the signal is rapidly lost, the attractors do not show a clear structure and cover the whole state-space. Additionally, the PSDs show broadband peaks with high energy levels at low frequencies. However, at low reduced velocities a single frequency could be identified in the PSD, but as the reduced velocity is increased distinct secondary frequencies appear (period-2 or even period-3 motion). All of this evidence suggest that the dynamics of this filament are always dominated by a random component throughout the reduced velocities tested.
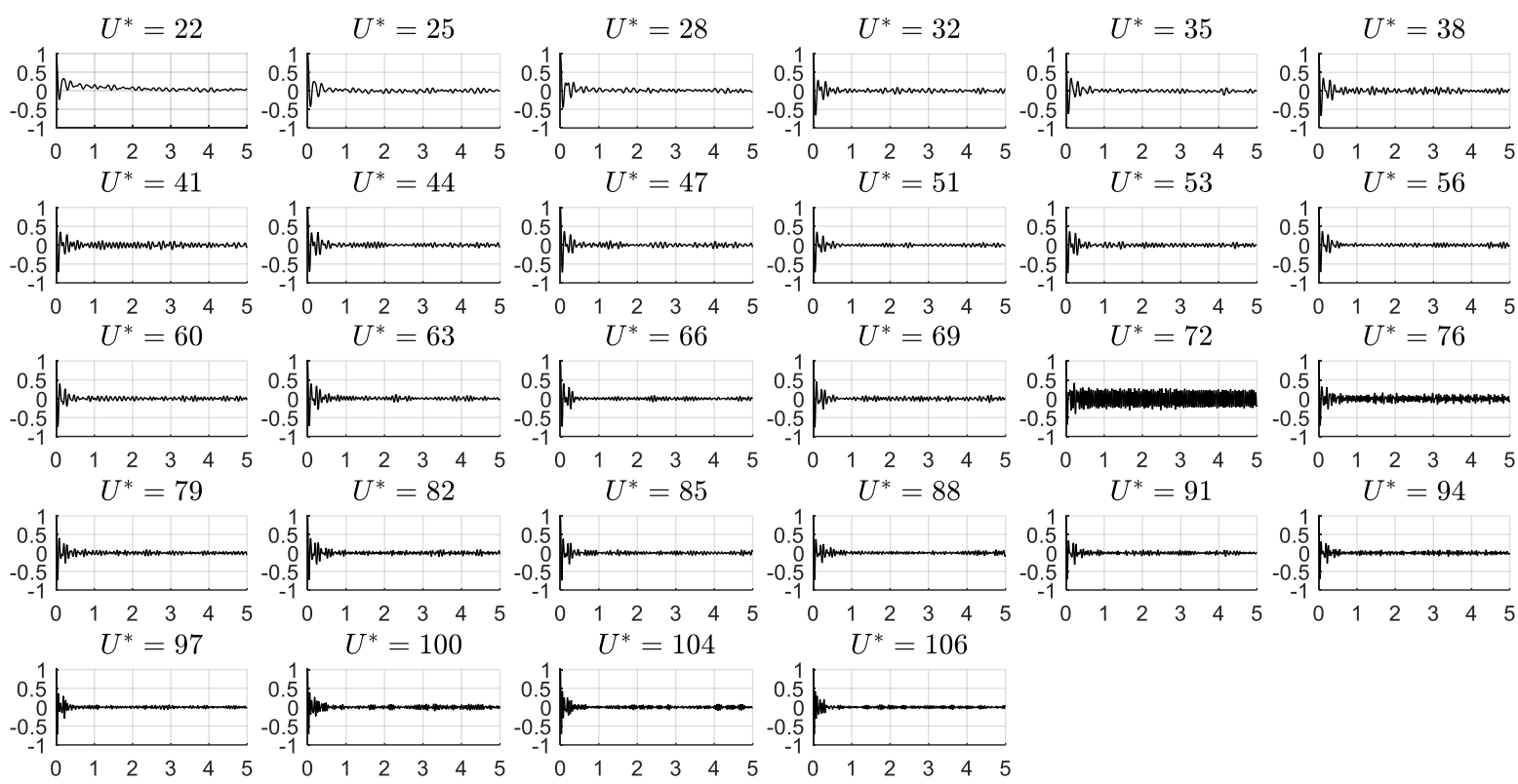

Figure 12. Autocorrelation function of the displacements of the free end of filament $3, \alpha=0^{\circ}$ (horizontal axis unit is $\mathrm{s}$ ). 


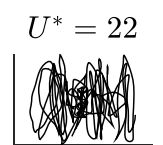

$U^{*}=41$

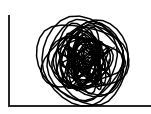

$U^{*}=60$

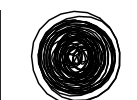

$U^{*}=79$



$U^{*}=97$

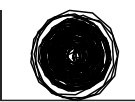

$U^{*}=25$

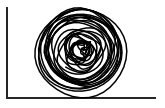

$U^{*}=44$

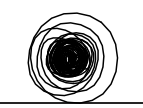

$U^{*}=63$



$U^{*}=82$

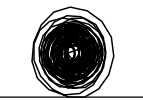

$U^{*}=100$

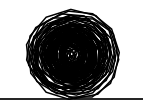

$U^{*}=28$

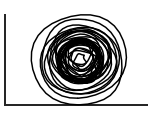

$U^{*}=47$

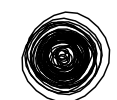

$U^{*}=66$

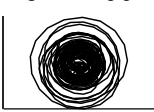

$U^{*}=85$

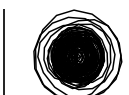

$U^{*}=104$

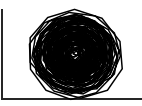

$U^{*}=32$

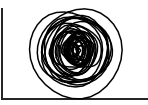

$U^{*}=51$

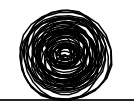

$U^{*}=69$

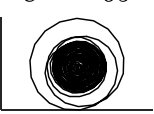

$U^{*}=88$

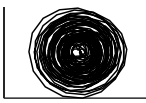

$U^{*}=106$



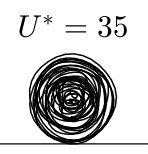

$U^{*}=38$

$U^{*}=53$

$U^{*}=56$
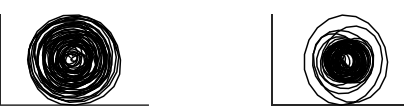

$U^{*}=72$

$U^{*}=76$
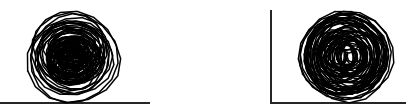

$U^{*}=91$

$U^{*}=94$
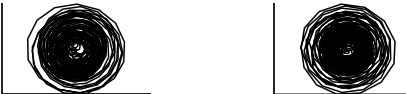

Figure 13. Reconstructed attractors in phase-space for the free end of filament $3, \alpha=0^{\circ}$. Horizontal axes: $r_{y z}(t)$, vertical axes: $r_{y z}(t+\tau)$.
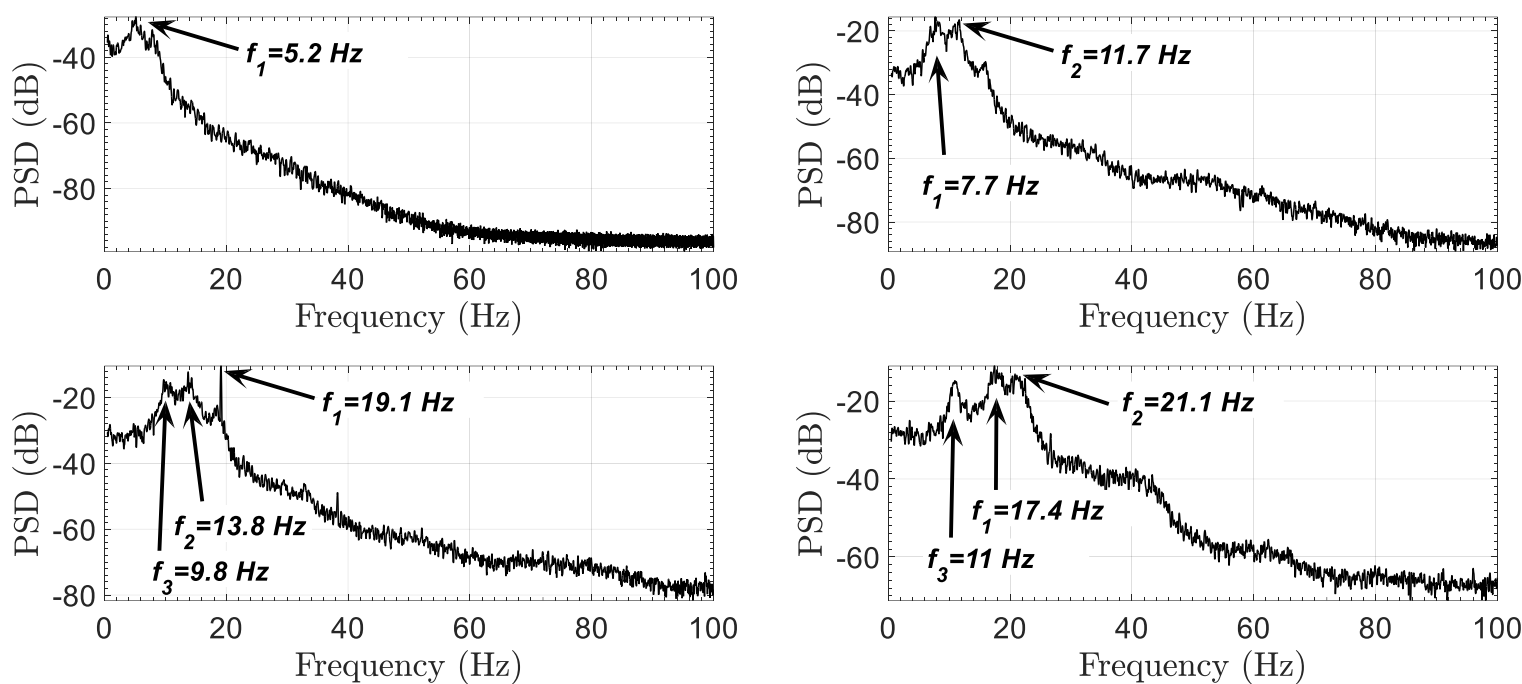

Figure 14. PSD of displacements of the free end of filament $3, \alpha=0^{\circ}$. (top left) $U^{*}=22$, (top right) $U^{*}=47$, (bottom left) $U^{*}=76$, (bottom right) $U^{*}=106$.

\subsubsection{Filament 1}

Finally, In Fig. 15 through 17 we show results for filament 1 when attached at $\alpha=90^{\circ}$. In Fig. 15 for the whole range of reduced velocities we observe short-decay correlations which suggest the motions of filament 1 are characterized by a high component of randomness. In Fig. 16 the reconstructed attractors in phase-space appear to be covered by the trajectories with no clear patterns. Moreover, the power spectra in Fig. 17 reveal that at low reduced velocities the timeseries is characterized by a single broadband frequency peak with high energy content at low frequencies, typical of chaotic motions. As the reduced velocity is increased the dominant peak in the power spectra becomes broader. Moreover, secondary frequencies emerge and coalesce into a broad peak at the highest reduced velocities tested. Together these plots reassure that this short 
filament is characterized by a period-1 motion with a high component of randomness throughout the range of reduced velocities tested. Noteworthy, these trends hold for all the cases regardless of the filament attachment angle. For this reason we did not carry out additional tests with this filament at intermediate attachment angles.
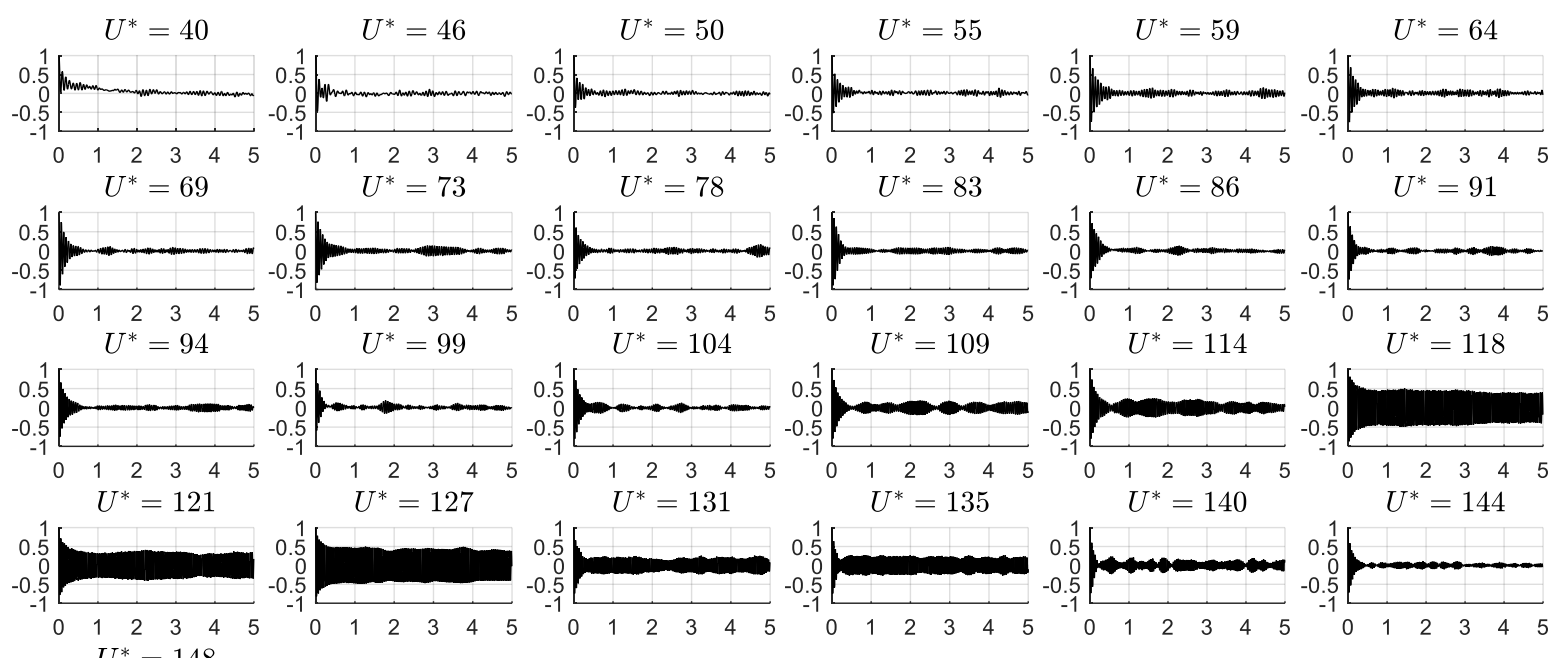

$U^{*}=135$

$U^{*}=140$

$U^{*}=144$

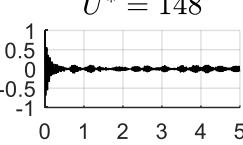

Figure 15. Autocorrelation function of the displacements of the free end of filament $1, \alpha=90^{\circ}$ (horizontal axes are delay time in seconds).
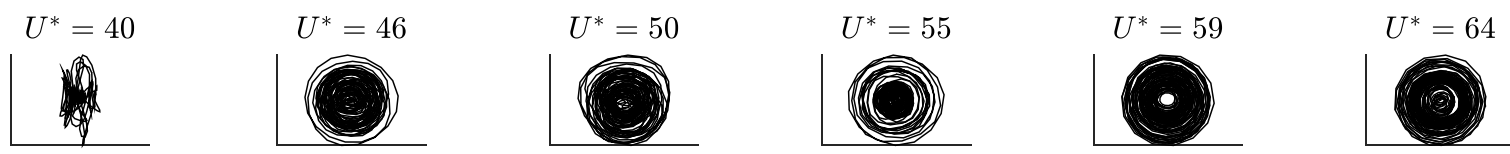

$U^{*}=69$

$$
U^{*}=73
$$

$$
U^{*}=78
$$

$$
U^{*}=83
$$

$$
U^{*}=86
$$

$U^{*}=91$


$U^{*}=121$
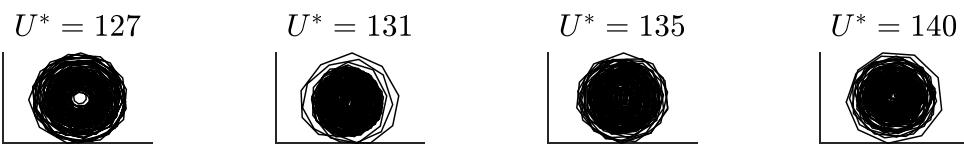

$U^{*}=148$
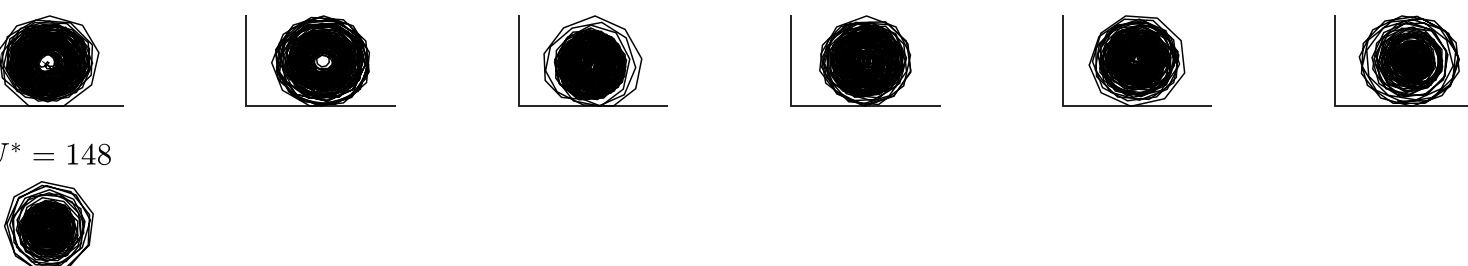

Figure 16. Reconstructed attractors in phase-space for the free end of filament 1, $\alpha=90^{\circ}$. Horizontal axes: $r_{y z}(t)$, vertical axes: $r_{y z}(t+\tau)$. 

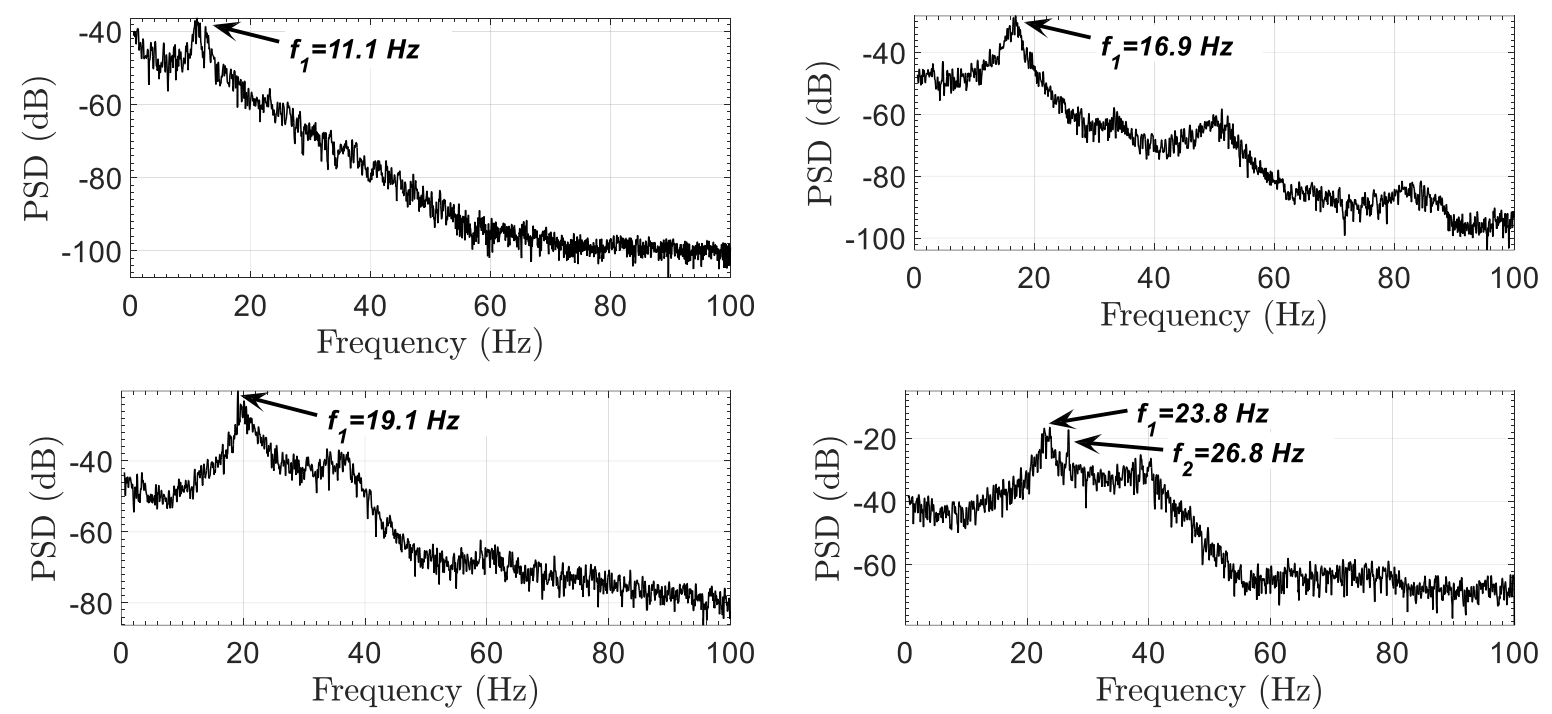

Figure 17. PSD of displacements of the free end of filament $1, \alpha=90^{\circ}$. (top left) $U^{*}=40$, (top right) $U^{*}=73$, (bottom left) $U^{*}=109$, (bottom right) $U^{*}=148$.

From the results presented, the main difference in the dynamics between filament 1 and filament 3 is the transition of filament 3 to a limit-cycle oscillation at a certain critical reduced velocity. However, when filament 3 is attached at angles $\alpha \lesssim 45^{\circ}$, it skips the LCO dynamics. Instead it behaves similar to filament 1 , i.e. exhibits a period-1 vibration at low velocities, which at higher reduced velocities bifurcates into period-2 or even period-3 motions with low frequency broadband peaks in the PSDs. In Fig. 8 we have shown the amplitude response plots where the red-filled symbols correspond to the data points where LCO-like dynamics was observed while the blackfilled symbols are for the intermittent LCO dynamics (ILCO). As can be seen, Filament 1 did not exhibit LCO dynamics regardless of the attachement angle. On the other hand, Filament 3 entered into LCOs at a certain reduced velocity and as long as $\alpha>45^{\circ}$, while for Filament 2 LCOs were observed for $\alpha \geq 45^{\circ}$.

As regards to the frequency of the motions these are represented in dimensionless form as $f_{y z}^{*}=$ $f_{y z} / f_{n 1}$ where $f_{y z}$ corresponds to the most prominent peak found from the power spectral density of frequencies at each reduced velocity, and $f_{n 1}$ is the first mode natural frequency of the filament. As can be seen in Fig. 18, $f_{y z}^{*}$ increases linearly with respect to the reduced velocity, where it can be appreciated that for each filament the data points tend to cluster around a single trend line. Noteworthy, the attachment angle has no effect on the flapping frequency of the filaments, and moreover the abrupt transitions from a noisy vibration to large-amplitude LCO or ILCO dynamics have no incidence either. Furthermore, it can be noted that the onset of resolvable motions (vibrations) occur around $U^{*} \approx 20-30$ where $f_{y z} \approx 2 f_{n 1}$. 

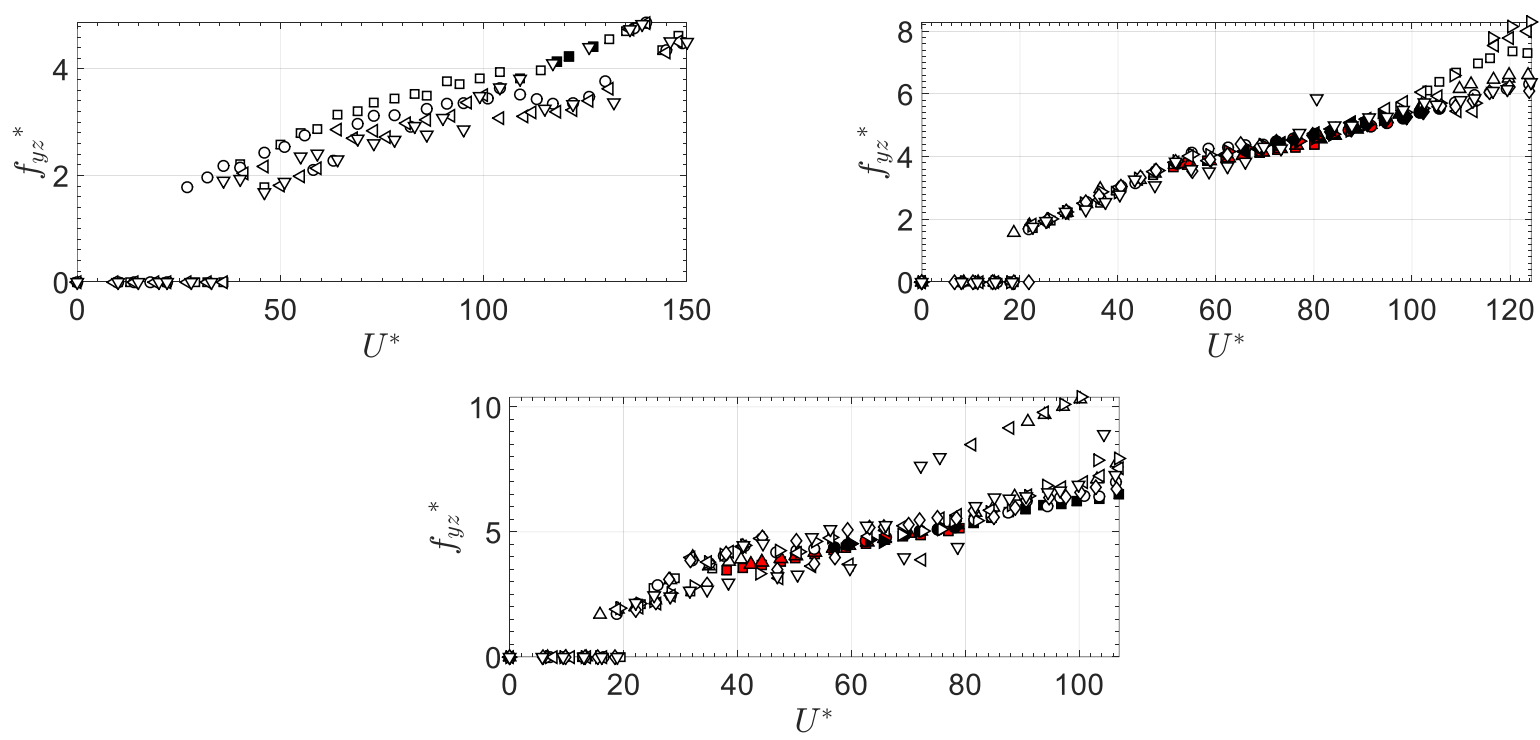

Figure 18. Dimensionless flapping frequency of filament 1 (top left), filament 2 (top right) and filament 3 (bottom) at different attachment angles $\alpha$ : $90^{\circ}(\square), 75^{\circ}(\triangle), 60^{\circ}(\circ), 45^{\circ}(\triangleright), 30^{\circ}(\triangleleft), 15^{\circ}(\diamond)$, and $0^{\circ}(\nabla)$. Red symbols: LCO; Black symbols: ILCO.

\subsection{Filaments dynamics interpretation}

In this section we discuss the possible mechanisms responsible for the observed filaments dynamics. In a previous study [9] turbulent buffeting was identified as the main source of excitation of flexible filaments. The requirement for this is that the integral turbulence length scale (macroscale) of the wind flow should be comparable to the size of the filament facing the flow, condition which is satisfied in the present experiments (see Fig. 3). In conclusion, the main source of excitation is turbulent buffeting.

In regards to the LCO and ILCO dynamics observed in several cases presented here, it is known that under the influence of extraneously-induced excitations a lightly damped structure may incur into large-amplitude self-excited motions while large damping prevents large-amplitude motions [17]. Therefore, the absence of LCO dynamics for filament 1 can be explained in terms of its relatively higher damping ratio compared to filaments 2 and 3 (lower damping), congruent with the findings reported by Silva-Leon et al [9] for a filament of similar dimensions ( $L=20 \mathrm{~mm}, D=0.61$ $\mathrm{mm}$ ). However, the level of damping does not appear to be the only requirement for determining the susceptibility of the filaments to enter into LCO dynamics. In fact, the absence of LCO dynamics for filament 2 and 3 at attachment angles $\alpha<45^{\circ}$ cannot be explained with this argument alone because preliminary free-vibration experiments (not documented here) revealed that the damping ratio in still air for these filaments remain unchanged as the attachment angle was varied between $90 \geq \alpha \geq 0^{\circ}$.

Besides these observations, the only feature that changes when varying $\alpha$ is the curvature of the localized bend of the filament at the fixed end. It might be thought that this localized curvature does not play a significant role on the flapping motions, considering the fact that the filaments possess a very low bending rigidity. As a matter of fact, the effect of the localized bend for filaments 2 and 3 was negligible when predicting their equilibrium reconfiguration angle (see Fig. 7). In fact, from a mechanical point of view these filaments may be modelled as a cantilever beam with an upstream torsion spring representing the mechanical effect of the localized bend. However, the direction and magnitude of the restoring elastic moment produced by the bend depend on how the filament hangs relative to its undeformed configuration (i.e. fully straight with no curvatures due to external loads). For example, as illustrated in Fig. 19 for the case of $\alpha=90^{\circ}$, when the filament is lifted by the fluid flow, the bend produces an internal moment $M$ which acts to restore the filament to its 
undeformed configuration. In this case a harmonic motion (LCO) can be sustained due to the opposing moments due to the elastic and fluid forces. On the other hand, when $\alpha=0^{\circ}$ the undeformed configuration is parallel to the flow, and the moment due to the bend of the reconfigured filament acts in favor of the fluid load so that the harmonic motion (LCO) is not observed. It is worth mentioning that the jump in amplitude observed in Fig. 8 for filaments 2 and 3 does not seem to be a vortex-shedding lock-in region because HWA measurements reveal that the vortex shedding frequency is 2-3 orders of magnitude larger than the filament vibration and natural frequency, so that there is no time-scale match which would suggest the possibility of a VIV lock-in (this was also discussed by [8], [9]).
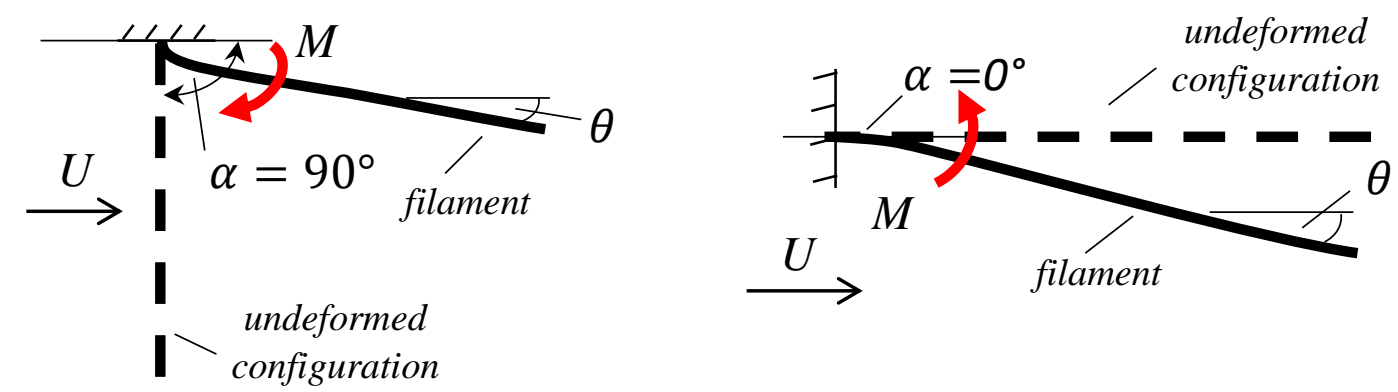

Figure 19. Moment direction due to bend for a filament when hanging at $\alpha=90^{\circ}$ (left) and $\alpha=0^{\circ}$ (right).

In summary, the noisy vibration dynamics is most likely produced by turbulent fluctuations which, at the onset of motion, excite the structure thus making it to oscillate at about twice the natural frequency of the filaments. Moreover, the localized moment generated by the small bend of the filaments plays a crucial role on the occurrence of LCO dynamics: if the elastic restoring moment acts against the moment due to the fluid load (which varies due to turbulent fluctuations) then harmonic oscillations can be sustained. Nevertheless, the susceptibility to enter into largeamplitude LCOs is also influenced by the damping ratio of the structure: if the filament is short enough then it will have a relatively higher damping ratio which reduces the vibrational motions and thus the possibility to enter into LCOs, at least within the range of wind velocities explored here. Consequently, under the conditions of the present experiments the bend, i.e. the attachment angle, also regulates the amplitude of the flutter motions and should not be disregarded.

In practical applications, it is important to determine the origin of flutter motions as it is known that the drag on a flexible structure significantly increases when it flutters [18] and this may reduce the effectiveness of vibrating or flapping structures for drag reduction applications [19]. In this regard, the results from the present investigation suggest that in certain systems flutter could be promoted or avoided if an appropriate attachment angle and boundary conditions are selected. These observations are critical for understanding of the performance and design of slender structures flapping in fluid flow, such as realistic hairy coatings or flags for energy harvesting. For instance, in a hairy coating, flexible filaments may be affixed to a curved surface (e.g. cylinder or wing profile) which would create various relative angles between the individual structures and the flow direction. As was shown, this could induce different responses which could affect the performance of a hairy coating. Moreover, in nature we can observe that flexible appendages, such as hairs or feathers, are not attached to a rigid surface but rather to a flexible substrate which may allow better bending behaviors and streamlining and thus reduces the reactive moment responsible of flutter motions. Therefore, we suggest future research to consider the effect of attachment angle on the dynamics of flexible fluid-structure interactions. 


\section{Concluding remarks}

We have investigated in a wind tunnel the motions of three cantilever flexible filaments of different aspect ratios $(L / D=50,100,150)$ which were held at various attachment angles $\left(0 \leq \alpha \leq 90^{\circ}\right)$ with respect to the direction of the incoming air flow in the range of reduced velocities of $7<U^{*}<150$. When subjected to the uniform flow the filaments were reconfigured thus leaving a fixed bent upstream end and free downstream end. The motions of the free end of the filaments were analysed via reconstructed attractors in phase space, autocorrelation functions and power spectral densities. Turbulent buffeting was identified as the main source of excitation of the filaments. It was found that even though the bending stiffness of the filaments is relatively low and the upstream bend is relatively small in length, its effect on the dynamical response is dramatic: for a certain range of attachment angles $\left(\alpha>45^{\circ}\right)$ the filaments exhibit small-amplitude vibrational motions at low reduced velocities, but at a certain reduced velocity the motion becomes large-amplitude characterized by limit-cycle oscillations (LCO) which progressively degraded at larger reduced velocities. For smaller attachment angles the filaments exhibit mostly small-amplitude vibrational motions throughout the range of reduced velocities tested. It was found that the range of reduced velocities at which large-amplitude LCOs were observed was gradually diminished and replaced by small-amplitude vibrational motions as the filament is attached at angles closer to the flow direction (i.e. closer to $\alpha=0^{\circ}$ ). It is suggested that the sustained harmonic oscillations (LCOs) are possible only if the reactive moment provided by the upstream bend of the filaments acts against the disturbing fluid load. A notable exception was the shortest filament which did not exhibit LCO dynamics, at least in the range of conditions tested. This was attributed to the higher damping level (double) compared to the larger filaments. Consequently, besides the structural damping related to the length, the boundary conditions (attachment angle) have dramatic effects on the dynamics of flapping structures in fluid flow and should be carefully taken into account in the study of flexible filaments in flow.

\section{Acknowledgments}

Jorge Silva-Leon gratefully acknowledges funding provided by SENESCYT through the scholarship Convocatoria Abierta 2012-I.

\section{Nomenclature}

$\begin{array}{cl}U & \text { Wind speed in the along wind direction (X direction) } \\ L & \text { Filament length } \\ D & \text { Filament diameter } \\ U^{*} & \text { Reduced velocity } \\ A^{*} & \text { Dimensionless amplitude of motion } \\ A & \text { Cross-section area } \\ A_{y z} & \text { Root-mean-square amplitude of motion in y and z directions } \\ r_{y z} & \text { Position time series of filament free end } \\ X, Y, Z \text { or } x, y, z & \text { X direction - along wind; Y direction - cross wind and parallel to gravity } \\ \zeta & \text { Damping ratio } \\ v & \text { Poisson ratio } \\ R e & \text { Reynolds number } \\ C a & \text { Cauchy number } \\ m^{*} & \text { Mass ratio } \\ B & \text { Buoyancy number } \\ E & \text { Modulus of elasticity } \\ E I & \text { Bending stiffness } \\ E(f) & \text { Power spectrum energy as function of frequency } \\ C_{d} & \text { Drag coefficient } \\ \rho_{f} & \text { Fluid density } \\ \rho_{s} & \text { Solid density (filament) }\end{array}$




$\begin{array}{cl}\theta & \text { Equilibrium angle in static reconfiguration } \\ \mu & \text { Dynamic viscosity } \\ \alpha & \text { Attachment angle } \\ L_{v x} & \text { Macroscale or turbulence integral length scale } \\ \Lambda_{v x} & \text { Microscale or dissipation length scale } \\ f_{n} & \text { Natural vibration frequency } \\ g & \text { Acceleration due to gravity } \\ M & \text { Moment due to localized bend of filament } \\ m & \text { Embedding dimension } \\ \tau & \text { Embedding delay } \\ t & \text { Time } \\ \bar{u} & \text { mean fluctuating velocity } \\ T u & \text { Turbulence intensity in the X direction }\end{array}$

\section{References}

[1] C. Grouthier, S. Michelin, R. Bourguet, Y. Modarres-Sadeghi, and E. de Langre, "On the efficiency of energy harvesting using vortex-induced vibrations of cables," $J$. Fluids Struct., vol. 49, pp. 427-440, 2014.

[2] S. G. Park, C. B. Chang, B. Kim, and H. J. Sung, "Simulation of fluid-flexible body interaction with heat transfer," Int. J. Heat Mass Transf., vol. 110, pp. 20-33, 2017.

[3] J. Favier, A. Dauptain, D. Basso, and A. Bottaro, "Passive separation control using a self-adaptive hairy coating," J. Fluid Mech., vol. 627, pp. 451-483, 2009.

[4] J. Niu and D. L. Hu, "Drag reduction of a hairy disk," Phys. Fluids, vol. 23, no. 10, p. $101701,2011$.

[5] H. Ait Abderrahmane, M. P. Paidoussis, M. Fayed, and H. D. Ng, "Flapping dynamics of a flexible filament," Phys. Rev. E, vol. 84, no. 6, p. 066604, 2011.

[6] L. Jia, Interaction between Flexible Plates and Fluid in Two-Dimensional Flow. Springer Berlin Heidelberg, 2014.

[7] J. Zhang, S. Childress, A. Libchaber, and M. Shelley, "Flexible filaments in a flowing soap film as a model for one-dimensional flags in a two-dimensional wind," Nature, vol. 408, no. 6814, pp. 835-839, Dec. 2000.

[8] L. Schouveiler, C. Eloy, and P. Le Gal, "Flow-induced vibrations of high mass ratio flexible filaments freely hanging in a flow," Phys. Fluids, vol. 17, no. 4, p. 047104, Apr. 2005.

[9] J. Silva-Leon, A. Cioncolini, A. Filippone, and M. Domingos, "Flow-induced motions of flexible filaments hanging in cross-flow," Exp. Therm. Fluid Sci., vol. 97, no. March, pp. 254-269, 2018.

[10] J. Silva-Leon, A. Cioncolini, and A. Filippone, "Determination of the normal fluid load on inclined cylinders from optical measurements of the reconfiguration of flexible filaments in flow," J. Fluids Struct., vol. 76, pp. 488-505, 2018.

[11] P. E. Roach, "The generation of nearly isotropic turbulence by means of grids," Int. J. Heat Fluid Flow, vol. 8, no. 2, pp. 82-92, 1987. 
[12] P. D. Welch, "The use of Fast Fourier Transform for the estimation of power spectra: a method based on time averaging over short, modified periodograms," IEEE Trans. Audio Electroacoust., vol. AU-15, no. 2, pp. 70-73, 1967.

[13] E. Bradley and H. Kantz, "Nonlinear time-series analysis revisited," Chaos, vol. 25, no. 9, 2015.

[14] H. Ait Abderrahmane, M. P. Païdoussis, M. Fayed, and H. Dick Ng, "Nonlinear dynamics of silk and Mylar flags flapping in axial flow," J. Wind Eng. Ind. Aerodyn., vol. 107-108, pp. 225-236, Aug. 2012.

[15] R. Hegger, H. Kantz, and T. Schreiber, "Practical implementation of nonlinear time series methods: The TISEAN package,” Chaos, vol. 9, no. 2, pp. 413-435, 1999.

[16] M. P. Paidoussis, Fluid-Structure Interactions: Slender Structures and Axial Flow, Volume 1, Second. Academic Press, 2014.

[17] E. Naudascher and D. Rockwell, Flow-Induced Vibrations: an Engineering Guide. Dover Publications, Inc., 1994.

[18] M. T. Morris-Thomas and S. Steen, "Experiments on the stability and drag of a flexible sheet under in-plane tension in uniform flow," J. Fluids Struct., vol. 25, no. 5, pp. 815830, 2009.

[19] T. Leclercq, N. Peake, and E. de Langre, "Does flutter prevent drag reduction by reconfiguration?," Proc. R. Soc. A Math. Phys. Eng. Sci., vol. 474, no. 2209, 2018. 


\section{Appendix}

Drag coefficient of a circular cylinder in pure crossflow

The drag coefficient versus Reynolds number correlation for smooth cylinders in cross-flow was derived in [10] and is reproduced for use here:

$$
C_{d}=\left(1.13+\frac{11.4}{R e^{0.808}}\right)^{0.952}
$$

where $R e=\rho_{f} U D / \mu$. The numerical coefficients were fitted to a large databank for cylinders in cross flow collected from the literature: 416 data points for smooth circular cylinders in cross flow covering Reynolds numbers between 0.1 to 300,000 . This fitted correlation has a Mean Absolute Percentage Error (MAPE) of $6.5 \%$ for $0.1<R e<500 ; 6.7 \%$ for $0.1<R e<1,000$; and, $7.3 \%$ for $0.1<$ $R e<300,000$. 